University of Nebraska - Lincoln

DigitalCommons@University of Nebraska - Lincoln

Norman R. Simon Papers

Research Papers in Physics and Astronomy

$6-1970$

\title{
CRITERIA FOR THE DISCOVERY OF BETA CEPHEI STARS ACCORDING TO THE MU -MECHANISM THEORY
}

Richard Stothers

Goddard Institute for Space Studies

Norman R. Simon

University of Nebraska - Lincoln, nsimon@unl.edu

Follow this and additional works at: https://digitalcommons.unl.edu/physicssimon

Stothers, Richard and Simon, Norman R., "CRITERIA FOR THE DISCOVERY OF BETA CEPHEI STARS ACCORDING TO THE MU -MECHANISM THEORY" (1970). Norman R. Simon Papers. 55.

https://digitalcommons.unl.edu/physicssimon/55

This Article is brought to you for free and open access by the Research Papers in Physics and Astronomy at DigitalCommons@University of Nebraska - Lincoln. It has been accepted for inclusion in Norman R. Simon Papers by an authorized administrator of DigitalCommons@University of Nebraska - Lincoln. 


\title{
CRITERIA FOR THE DISCOVERY OF BETA CEPHEI STARS ACCORDING TO THE MU-MECHANISM THEORY
}

\author{
RICHARD STOTHERS AND NORMAN R. SIMON \\ Goddard Institute for Space Studies \\ NASA, New York
}

Received March 19, 1.970

\begin{abstract}
Observational discriminants are proposed to test the $\mu$-mechanism theory of $\beta$ Cephei stars as advanced by Stothers and Simon (1969). These include: helium and nitrogen overabundances, far ultraviolet flux excesses (high surface temperature), narrow (occasionally broad) spectral line widths, and binary character. A list of potential $\beta$ Cep candidates, comprising 24 spectroscopic binaries with spectral type O9-B4 III-V and a large orbital ratio of masses, has been compiled for the benefit of observers interested in searching for new $\beta$ Cep stars. The data thus far accumulated are not in contradiction with, and occasionally positively support, the $\mu$-mechanism theory.
\end{abstract}

\section{Introduction}

In a recent paper (Stothers and Simon 1969) we proposed the following theory of $\beta$ Cephei stars. In a close binary system the original primary star, being more massive than the secondary, will evolve first, expanding its radius during hydrogen burning until it reaches its Roche lobe. Thereupon it will quickly lose mass to the secondary, which accretes first the outer zero-age envelope of the primary and then, on top of that, the helium-enriched outer portions of the primary's core. If sufficient helium is accreted and the total mass exceeds $6 \mathfrak{M}_{\odot}$, the compression of the secondary's envelope by the weight of the helium ( $\mu$ mechanism) has been found to raise the effective temperature and to permit the development of radial pulsations which are strongly energized by the hydrogenburning reactions in the core. Considerable observational evidence in favor of this theory can be found in our original paper, to which we refer the reader for details not covered in the following.

Recent lists of the "classical" $\beta$ Cep stars, with confirmed periodic variations in light and radial velocity, can be found in papers by Hill (1967), Leung (1967), McNamara and Matthews (1967), and Percy (1970). Earlier lists are referenced, for example, by Hill and Leung. Additional numbers of suspected $\beta$ Cep stars have been 
tabulated or referred to by Petrie and Pearce (1962), Hill (1967), and Percy (1970), but the properties of these suspects have yet to be confirmed.

In the interest of uncovering additional members of the $\beta$ Cep group with the particular aim of further testing the validity of our theory, we list the most basic observable properties of potential $\beta$ Cep stars as (1) apparent MK spectral type and luminosity class $\mathrm{O}-\mathrm{B} 3 \mathrm{II}-\mathrm{V}$; (2) short-period variations in light and radial velocity; (3) sharp (or broad) spectral lines; (4) binary character with a large mass ratio; (5) helium and nitrogen overabundance; and (6) far ultraviolet flux excess. Individually, these criteria are necessary but not sufficient conditions for $\beta$ Cep stars. Since criteria (3), (4), (5), and (6) refer specifically, albeit not uniquely, to our theory, it is these which we shall now consider in detail.

\section{Helium and Nitrogen Abundances}

The expected helium overabundance in our theory may be difficult to detect observationally because only little surface helium enrichment is sufficient to destabilize our models. Thus, the fractional abundance of helium by mass at the surface of our models can be as low as $Y=0.6\left(15 \mathfrak{M}_{\odot}\right)$ and $Y=0.5\left(30 \mathfrak{M}_{\odot}\right)$. In this connection, we note that these limits on $Y$ may actually be lower still, because we overestimated (deliberately) the pulsational damping in our chemically unmixed models, and because we have subsequently discovered (Stothers and Simon 1970) that the faintest possible luminosity for chemically mixed models must be revised to about $M_{v}=-4.5$-too bright to explain most of the $\beta$ Cep stars. Thus the assumption of slow interior mixing has to be adopted if our theory is correct, and this permits a moderately low surface helium content in our models for the fainter $\beta$ Cep stars (as is actually observed).

Detailed abundance analyses are available for two $\beta$ Cep stars, $\gamma$ Pegasi and $\delta$ Ceti (Neven and de Jager 1954; Aller and Jugaku 1959), both of which seem to show "normal" helium with respect to other early B stars. However, such analyses for .O and B stars are now considered to be extremely unreliable concerning the helium abundance (see Underhill 1966). Moreover, it is uncertain how much helium has diffused below the photosphere, since the classical $\beta$ Cep stars, like $\gamma$ Peg and $\delta$ Cet, are very slow rotators, and other slowly rotating $B$ stars seem to show noticeable helium 
deficiencies (Hardorp and Strittmatter 1968). Therefore, the "normal" helium abundance which is observed in $\beta$ Cep stars may actually represent an overabundance. Good model atmospheres are essential to verify this point.

We also predict large N/C and N/O ratios at the surface, since the secondary products of old hydrogen burning should also be present. This has yet to be verified.

\section{Far Ultraviolet Fluxes}

In addition to chemical composition anomalies, the atmospheres of $\beta$ Cep stars are expected, on our theory, to be characterized by large values of $\log g=4.3-4.5$ and $T_{e}=40,000-50,000^{\circ} \mathrm{K}$. Although these temperatures are hotter than the ones normally associated with early B stars, the helium overabundance may alter the spectrum in the right direction (see Böhm-Vitense 1967). As a possibly relevant example of this, Underhill (1966) finds the classical helium star HD.96446. to be of type $\mathrm{O}$ with a shell spectrum, but Buscombe (1965), in a different examination, finds it to be of type Bl V. In any event, the expected ultraviolet excess of our $40,000^{\circ} \mathrm{K}$ models over "normal" $30,000^{\circ} \mathrm{K}$ models of $\beta$ Cep stars in the currently measured wavelength range $\lambda 1115-\lambda 3000$ will be detected only with difficulty because of the relatively small changes of flux with temperature at very high temperatures, the deep line blanketing of the ultraviolet continuum, and the selective interstellar extinction which becomes large in the far ultraviolet. Thus, the fact that the measured ultraviolet color indices for known $\beta$ Cep stars scatter around those for nonvariables of spectral type late $\mathrm{O}$ to early B (Byram, Chubb, and Werner 1965; Smith 1967; Bless, Code, and Houck 1968; Stecher 1969) should not be considered meaningful. Furthermore, Stecher points out that a (possible) ten percent error in the observed ultraviolet flux at $\lambda 2000$ for a star of effective temperature $40,000^{\circ} \mathrm{K}$ implies an error in the effective temperature of $10,000^{\circ} \mathrm{K}$.

High absolute photometric accuracy, coupled with detailed model atmospheres, is unquestionably required to obtain a definitive effective temperature scale for hot stars.

\section{Spectral Line Width}

The presence of sharp spectral lines in a star may indicate intrinsically slow rotation or a small angle of inclination of the line 
of sight to the axis of rotation. All of the classical $\beta$ Cep stars with measured rotational velocities show abnormally sharp lines for their spectral and luminosity classifications, which has led to the frequent suspicion that they are all intrinsically slow rotators and possibly all members of binary systems. Analysis of the light and radial-velocity curves of a number of these variables has, in fact, substantiated the fact that some are the brighter components of spectroscopic binary systems (e.g., Batten 1967; Fitch 1967, 1969). All of the known $\beta$ Cep members of binaries exhibit a long spectroscopic "beat" period, which, in the case of every $\beta$ Cep star showing this phenomenon, may be due simply to orbital motion. This possibility has been suggested independently by Shobbrook et al. (1969), who discovered that the brighter component of the wellknown spectroscopic and eclipsing binary $\alpha$ Virginis is a $\beta$ Cep star.

Broad spectral lines, of course, are not incompatible with membership in a binary system. For example, $\alpha$ Vir exhibits broad lines (high rotation). A number of suspected $\beta$ Cep stars also show the normal broad lines of $\mathrm{B}$ giants as well as long-period changes in radial velocity, which may be due to orbital motion (e.g., Breger 1967).

No searches for $\beta$ Cep stars have been carried out solely on the basis of the width of spectral lines, and it is now clear that none should be.

\section{Binaries}

At the present time, we are reduced to using known spectroscopic binaries with large mass ratio as potential candidates for $\beta$ Cep stars according to our theory. One thinks of Wolf-Rayet binary systems, but the evolved and less massive (Wolf-Rayet) component is normally the brighter member and fluctuates irregularly in light, so that, even if the secondary has accreted sufficient helium to become pulsationally unstable, its pulsations would be hard to detect. Instead, we have concentrated on the other $\mathrm{O}$ and B spectroscopic binaries, listed in Batten's (1967) Sixth Catalogue of the Orbital Elements of Spectroscopic Binary Systems. Our selections will, of course, depend heavily on the (occasionally uncertain) reliability of the available mass ratios and spectral types, which have been chosen according to the criteria: spectral type O9-B4 II-V for the more massive star (the potential $\beta$ Cep candidate) and 
$\mathfrak{M}_{1} \sin ^{3} i>>\mathfrak{M}_{2} \sin ^{3} i$. Table I lists the selected systems, many of which are eclipsing, with the following data.

Columns 1 and 2: HD number and name.

Columns 3 and 4: apparent visual magnitude (photographic magnitudes in parentheses) and orbital period in days, both from Batten (1967).

Columns 5 and 6: spectral type and luminosity class of the brighter and fainter components (MK classes when available), from Jaschek, Conde, and de Sierra (1964), Sahade (1962), Slettebak (1963), Batten (1967), or Olson (1968).

Columns 7 and 8: $\mathfrak{M} \sin ^{3} i$ (in solar units) of the brighter and fainter components, from Batten (1967) or Sahade (1962). True masses, corrected for orbital inclination, are shown underlined.

Columns 9 and 10: apparent rotational velocity, $v \sin i$ (in $\mathrm{km} /$ sec), of the brighter and fainter components, from Boyarchuk and Kopylov (1964), Slettebak (1956, 1963, 1966, 1968), Koch, Olson, and Yoss (1965), or Hill (1967).

Column 11: our classification of the system. In class-A systems, the star with smaller mass is clearly more evolved (has a brighter luminosity class) than its companion, or else has the same or an earlier MK spectral type. Class-B systems are similar to class-A systems except that $\mathfrak{M \ell _ { 1 }}<10 \mathfrak{M}_{\odot}$. (Our theoretical models indicate that stars with a mass as low as $6 \mathfrak{M}_{\odot}$ can be destabilized, but the only likely $\boldsymbol{\beta}$ Cep candidates are stars with masses greater than $\sim 10 \mathfrak{M}_{\odot}$.) In class-C systems, the spectral type of the star with smaller mass is uncertain, but is probably the same as or earlier than the spectral type of its companion. Class-D systems comprise the remainder of the stars, by analogy with $\alpha$ Vir which does not fit into class $\mathrm{A}, \mathrm{B}$, or $\mathrm{C}$.

The available data indicate that, in some cases, the mass of the brighter star is much larger (or smaller) than that normally associated with its spectral type. Could the B spectral type be an effect of helium overabundance, as suggested above? It is worth noting, in this connection, that all of the "helium" white dwarfs are found in an analogously narrow range of color index and spectral type (Eggen and Greenstein 1965). Accurate light and radial-velocity curves of the most anomalous systems in our list may permit the detection of not only $\beta$ Cep variability but also the small radii (for 
TABLE I

Early-Type Spectroscopic Binaries With a Large Mass Ratio

\begin{tabular}{|c|c|c|c|c|c|c|c|c|c|c|}
\hline HD & Name & $m$ & $\begin{array}{c}P \\
\text { (days) }\end{array}$ & $\mathrm{Sp}_{1}$ & $\mathrm{Sp}_{2}$ & $\underset{\left(M_{i} \sin ^{3} i\right.}{\left.M_{i}\right)}$ & 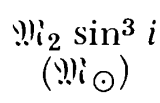 & $\begin{array}{c}v_{1} \sin i \\
(\mathrm{~km} / \mathrm{sec})\end{array}$ & $\begin{array}{c}v_{2} \sin i \\
(\mathrm{~km} / \mathrm{sec})\end{array}$ & Class \\
\hline 3369 & $29 \pi$ And & 4.4 & 143.6 & B5 V & B3 & 26.9 & 10.9 & 33 & & A \\
\hline 10516 & $\phi$ Per & 4.1 & 126.6 & $\mathrm{~B} 2 \mathrm{Ve}$ & & 15 & 7.5 & 450 & & $\mathrm{D}^{\circ}$ \\
\hline 19820 & CC Cas & $(7.0-7.1)$ & 3.4 & O9 IV & O9 IV & 18.9 & 9.2 & & & A \\
\hline 23180 & 38 o Per & 3.8 & 4.4 & B1.5 IV-III & $\mathrm{B} 2 \mathrm{~V}$ & 5.2 & 3.6 & $75:$ & 150: & $\mathrm{D}^{\circ}$ \\
\hline 25204 & $35 \lambda$ Tau & $3.4-3.9$ & 4.0 & B3 V & A4 IV & $\underline{6.1}$ & $\underline{1.6}$ & 117 & & $\mathrm{~B}^{\circ}$ \\
\hline 29763 & $94 \quad \tau$ Taul & 4.3 & 3.0 & B3 V & Al V & $\overline{3.0}$ & $\overline{0.9}$ & 150 & 100 & $\mathrm{D}$ \\
\hline 33357 & SX Aur & $(8.4-9.2)$ & 1.2 & B3 V & $\mathrm{B} 3 \mathrm{~V}$ & 11.2 & $\underline{5.9}$ & & & A \\
\hline 35715 & $30 \psi$ Ori & 4.6 & 2.5 & B2 IV & B0 & 8.7 & 5.3 & 166 & & $\mathrm{C}^{\circ}$ \\
\hline 37043 & $44 \imath$ Ori & 2.8 & 29.1 & O9 III & B3p & 15.9 & 9.4 & 100 & 50 & $\mathrm{D}$ \\
\hline 37756 & & 4.9 & 27.2 & B3 III & $\mathrm{Bl}$ & 6.4 & 4.1 & 87 & & $\mathrm{C}^{\circ}$ \\
\hline 39698 & 57 Ori & 5.9 & 7.8 & $\mathrm{~B} 2 \mathrm{~V}$ & B5 & 10.3 & 3.9 & 140 & & $\mathrm{D}$ \\
\hline 42933 & $\delta \mathrm{Pic}$ & $4.7-4.9$ & 1.7 & B0.5 III & B0.5-3 & 16.3 & 7.3 & 270 & & $\mathrm{D}^{\circ}$ \\
\hline 65818 & V Pup & $4.1-4.7$ & 1.5 & Bl V & B3 IV & $\overline{18}$ & $\underline{10}$ & 180 & & A \\
\hline 104337 & & 5.3 & 3.0 & B1.5 V & & 8.2 & 4.4 & 143 & & D \\
\hline 116658 & $67 \alpha \mathrm{Vir}$ & $1.0-1.1$ & 4.0 & B2 V & B3 V & $\underline{9}$ & $\underline{5}$ & 172 & & $\mathrm{D}^{\circ}$ \\
\hline 121263 & $\zeta$ Cen & 2.5 & 8.0 & Bl-2 IV & & $\overline{6} .4$ & $\overline{4} .4$ & 200 & & $\mathrm{D}$ \\
\hline 144217 & $8 \beta \mathrm{Sco}$ & 2.6 & 6.8 & B0.5 V & B2 V & 16.0 & 9.6 & 85 & 85 & D \\
\hline 151890 & $\mu^{1}$ Sco & $3.0-3.3$ & 1.4 & $\mathrm{~B} 1.5 \mathrm{~V}$ & B6 & 14 & 9 & 220 & & D \\
\hline 156633 & $68 \mathrm{u}$ Her & $(4.5-5.2)$ & 2.1 & B3 III & B5 & $\overline{8.6}$ & $\underline{1} .3$ & 119 & 90 & $\mathrm{D}$ \\
\hline 173787 & V356 Sgr & $(7.0-8.0)$ & 8.9 & B3 V & A2 II & 12.1 & 4.7 & & & A \\
\hline 181987 & Z Vul & $8.0-9.0$ & 2.5 & B4 V & A2-3 III & $\overline{5.4}$ & $\overline{2.3}$ & & & B \\
\hline 187879 & V380 Cyg & $5.5-5.6$ & 12.4 & BI III & B3 V & $1 \overline{4.1}$ & $\overline{8.0}$ & 53 & & $\mathrm{D}^{\circ}$ \\
\hline
\end{tabular}


TABLE I (Continued)

\begin{tabular}{|c|c|c|c|c|c|c|c|c|c|c|}
\hline HD & Name & $m$ & $\begin{array}{c}P \\
\text { (days) }\end{array}$ & $\mathrm{Sp}_{1}$ & $\mathrm{Sp}_{2}$ & $\begin{array}{c}\left(! M_{1} \sin ^{3}\right. \\
\left(M_{\odot}\right)\end{array}$ & $\begin{array}{c}\left(M_{i} \sin ^{3} i\right. \\
\left(M_{i_{\odot}}\right)\end{array}$ & $\begin{array}{c}v_{1} \sin i \\
(\mathrm{~km} / \mathrm{sec}\end{array}$ & $\begin{array}{c}v_{2} \sin i \\
(\mathrm{~km} / \mathrm{sec})\end{array}$ & Class \\
\hline$+40^{\circ} 4220$ & V729 Cyg & $(10.0-10.3)$ & 6.6 & O9 & O7f & 7.8 & 1.9 & & & C \\
\hline 208095 & & 5.5 & 17.3 & B7 & B9 & 20.8 & 13.6 & & & A \\
\hline 209481 & 14 Cер & 5.6 & 3.1 & O9 V & O9 & 5.8 & 2.9 & & & $\mathrm{C}$ \\
\hline 214240 & & 6.3 & 10.9 & B3 V & B5 & 6.0 & 3.9 & 60 & & $\mathrm{D}$ \\
\hline 217312 & & 7.4 & 15.3 & BO IV & BO IV & 270 & 127 & 70 & & \\
\hline
\end{tabular}

${ }^{\circ}$ Notes to Table I

HD 10516: possibly variable in light with a short period (Lynds 1959).

HD 23180: variable in light with a short period according to Walker (1962), but probably constant according to Lynds (1959). Jones (1960) found variability in the radial-velocity curve.

HD 25204: a close triple system.

HD 35715: possibly variable in light with a short period according to Lynds (1959) and Hill (1967), the latter of whom called the star tentatively a $\beta$ Cephei star.

HD 37756: variable in light with a short period according to Lynds (1959), but constant according to Hill (1967).

HD 42933: variable in light with a short period according to Cousins (1966).

HD 116658: $\beta$ Cephei light curve (Shobbrook et al. 1969).

HD 187879: constant in light according to Hill (1967).

HD 217312: constant in light according to Hill (1967). 
their masses) of stars which have accreted an overabundance of helium.

Our list by no means exhausts the possible candidates for $\beta$ Cep stars. A few other binaries in the spectral range B5-B9 have large $\mathfrak{M}_{1}$, e.g., U Cep, $\zeta$ Phoenicis, $\boldsymbol{\beta}$ Persei, U Sagittae, $\pi$ Andromedae, and HD 208095. In fact, the last two stars have been included in our list because of their very large $\mathfrak{M}_{1}$. It is also possible that some systems with components having nearly equal spectral types and masses may have previously suffered extensive mass exchange. And some systems with a small mass function, but an invisible (hot) companion, may be even more likely candidates for having exchanged mass (our proposal for the already known $\beta$ Cep stars). Finally, we should caution that most of the systems in class D probably consist of normal pairs of main-sequence stars, and that, unless the secondary in any system which has suffered mass exchange accretes sufficient helium, it would not be expected to exhibit $\beta$ Cep behavior.

One of us (R.S.) thanks Drs. K.-C. Leung and N. J. Woolf for conversations leading to the preparation of this paper. The second writer (N.R.S.) acknowledges the support of a NAS-NRC Research Associateship under the National Aeronautics and Space Administration.

\section{REFERENCES}

Aller, L. H., and Jugaku, J. 1959, Ap. J. Suppl. 4, 109.

Batten, A. H. 1967, Pub. Dominion Astrophys. (Obs. 13, 119.

Bless, R. C., Code, A. D., and Houck, T. E. 1968, Ap. J. 153, 561.

Böhm-Vitense, E. 1967, Ap. J. 150, 483.

Boyarchuk, A. A., and Kopylov, I. M. 1964, Izu. Crimean Ap. (Obs. 31, 44.

Breger, M. 1967, M.N.R.A.S. 136, 51.

Buscombe, W. 1965, M.N.R.A.S. 129, 1.

Byram, E. T., Chubb, T. A., and Werner, M. W. 1965, Ann. d'Ap. 28, 594.

Cousins, A. J. 1966, M.N.R.A.S. 131, 443.

Eggen, O. J., and Greenstein, J. L. 1965, Ap. J. 141, 83.

Fitch, W. S. 1967, Ap. J. 148, 481.

- 1969, Ap.J. 158, 269.

Hardorp, J., and Strittmatter, P. A. 1968, Ap. J. 153, 465.

Hill, G. 1967, Ap.J. Suppl. 14, 263.

Jaschek, C., Conde, H., and Sierra, A. C. de 1964, La Plata Ser. Astr. 28 (2). Jones, D. H. P. 1960, M.N.R.A.S. 120, 43.

Koch, R. H., Olson, E. C., and Yoss, K. M. 1965, Ap. J. 141, 955.

Leung, K.-C. 1967, Ap. J. 150, 223. 
Lynds, C. R. 1959, Ap. J. 130, 577.

McNamara, D. H., and Matthews, G. E. 1967, in Modern Astrophysics, M. Hack, ed. (Paris: Gauthier-Villars), p. 127.

Neven, L., and de Jager, C. 1954, B.A.N. 12, 103.

Olson, E. C. 1968, Ap. J. 153, 187.

Percy, J. R. 1970, Ap. J. 159, 177.

Petrie, R. M., and Pearce, J. A. 1962, Pub. Dominion Astrophys. (Obs. 12, 1. Sahade, J. 1962, in Symposium on Stellar Evolution, J. Sahade, ed. (La Plata: Observatorio Astronómico, Universidad Nacional de La Plata), p. 185.

Shobbrook, R. R., Herbison-Evans, D., Johnston, I. D., and Lomb, N. R. 1969, M.N.R.A.S. 145, 131.

Slettebak, A. 1956, Ap. J. 124, 173.

— 1963, Ap. J. 138, 118.

- 1966, Ap.J. 145, 126.

— 1968, Ap.J. 151, 1043.

Smith, A. 1967, Ap.J. 147, 158.

Stecher, T. P. 1969, A.J. 74, 98.

Stothers, R., and Simon, N. R. 1969, Ap. J. 157, 673.

- $1970, A$ p. J. 160 (in press).

Underhill, A. B. 1966, The Early Type Stars (Dordrecht, Holland: D. Reidel Publishing Co.).

Walker, M. F. 1952, A.J. 57, 227. 